\title{
Investigations on the TOPSAR acquisition mode with TerraSAR-X
}

\author{
Adriano Meta, Josef Mittermayer, Ulrich Steinbrecher and Pau Prats \\ German Aerospace Center, Microwave and Radar Institute \\ P.O. Box 1116, 82230 Wessling, Germany, Email: adriano.meta@dlr.de
}

\begin{abstract}
The paper reports about investigations on the implementation of the recently proposed TOPSAR acquisition mode with TerraSAR-X. TOPSAR mode allows a wide swath coverage with nearly uniform signal to noise ratio. To achieve this goal the antenna beam is steered within the different swaths in the along-track direction. TerraSAR-X can electronically steer the azimuth beam; however, angle quantization is introduced because of the limited number of available azimuth beams per data take. The effects of the TerraSAR-X angle quantization of the antenna steering are analyzed resulting in a negligible distortion. The maximum azimuth steering angle of TerraSAR-X allows the implementation of TOPSAR with the same coverage and resolution as the nominal TerraSAR-X ScanSAR mode, four range subswaths with $16 \mathrm{~m}$ azimuth resolution. Finally, TerraSAR-X TOPSAR interferometry capability and the possibility of using an inverse TOPSAR configuration are discussed.
\end{abstract}

\section{INTRODUCTION}

TerraSAR-X is a versatile SAR satellite which is able to perform SAR acquisition in different modes: stripmap, sliding spotlight, ScanSAR. In ScanSAR, azimuth resolution is traded for swath coverage. The increased range coverage is obtained by switching the antenna elevation beam in order to illuminate a larger area in the cross-track direction [1]. To achieve this goal, the target azimuth illumination is reduced when compared to the stripmap mode. In the burst processing, different parts of the antenna pattern are used to focus the burst images. The consequence of this approach is, in addition to a reduced azimuth resolution, the presence of scalloping and azimuth dependent ambiguity performance. The azimuth resolution of the standard TerraSAR-X ScanSAR image is $16 \mathrm{~m}$, covering approximately $120 \mathrm{~km}$ in range with four subswaths.

TerraSAR-X is able to electronically steer the antenna azimuth pattern. This capability offers the opportunity to implement on the satellite different acquisition modes for wide swath coverage, i.e. the recently published TOPSAR acquisition mode [2]. In both ScanSAR and TOPSAR, the azimuth resolution reduction is caused by a shorter target illumination. In ScanSAR this is achieved by illuminating targets with only a small portion of the antenna pattern; in TOPSAR the shorter target illumination time $T_{D}$ is obtained by sweeping the antenna from back to forth, in an opposite fashion as in spotlight. A nearly constant signal to noise ration (SNR) and distributed target ambiguity ratio (DTAR) in the azimuth direction are obtained.
In TOPSAR the resolution loss is achieved by shrinking the antenna footprint, rather than slicing it, as it happens for ScanSAR. The Doppler bandwidth acquired for each target in the swath is no longer directly related only to the burst time $T_{B}$, but also to the antenna steering angle rate $k_{\phi}$. Assuming a sinc squared azimuth pattern, the resulting TOPSAR antenna pattern is [2]:

$$
\begin{aligned}
G_{T}(\phi(\tau)) & \approx G_{0} \operatorname{sinc}^{2}\left(\frac{L}{\lambda}\left(\frac{v \tau}{R_{0}}+\phi(\tau)\right)\right) \\
& =G_{0} \operatorname{sinc}^{2}\left(\frac{L v \tau}{\lambda R_{0}}\left(1+\frac{R_{0} k_{\phi}}{v}\right)\right)
\end{aligned}
$$

where $\phi(\tau)$ is the antenna rotation angle as function of the slow time $\tau$. The resulting TOPSAR azimuth resolution is shrunk with respect to the stripmap resolution by a factor $\alpha$ equal to:

$$
\alpha=1+\frac{R_{0} k_{\phi}}{v}
$$

leading to:

$$
\rho_{a z}=\rho_{a z_{S T R I P}} \alpha=\frac{\lambda}{2 \phi_{0}}\left(1+\frac{R_{0} k_{\phi}}{v}\right)
$$

where $\phi_{0}$ is antenna azimuth beamwidth exploited for focusing the SAR data. The choice of $\phi_{0}$ is a tradeoff between resolution (requiring large interval) and ambiguity suppression and SNR (requiring small interval). Different values can be chosen for the different subswaths. Once the value of $\phi_{0}^{n}$ is fixed (the apex $n$ refers to the $n^{\text {th }}$ subswath), the steering angle parameters can be derived by inverting (3) [2]:

$$
k_{\phi}^{n}=\left(\frac{2 \rho_{a z} \phi_{0}^{n}}{\lambda}-1\right) \frac{v}{R_{0}^{n}}
$$

After the steering angle rates have been calculated, the global TOPSAR time line can be derived imposing that no gaps are left in the covered area. This constraint results in a set of $n$ linear equations [2]:

$$
\left(k_{\phi}^{n} T_{B}^{n}-\phi_{0}^{n}\right) R_{0}^{n}+v T_{B}^{n}=v T_{R}
$$

where $T_{R}=\sum_{n} T_{B}^{n}+T_{G}$ is the cycle time and $T_{G}$ is a time margin that can be used to assure a sufficient overlapping of two bursts in the same subswath. Finally, having the time line and the steering angle rates, it is possible to calculate the maximum steering angle required per each subswath. 
TABLE I

TERRASAR-X PARAMETERS

\begin{tabular}{|c|c|}
\hline Carrier frequency & $9.65 \mathrm{GHz}$ \\
\hline Azimuth beamwidth $(3 \mathrm{~dB})$ & $0.33^{\circ}$ \\
\hline Ground velocity & $6800 \mathrm{~m} / \mathrm{s}$ \\
\hline Maximum steering capability & $\pm 0.75^{\circ}$ \\
\hline Height & $514 \mathrm{~km}$ \\
\hline
\end{tabular}

An example of TOPSAR time line calculation is reported using the TerraSAR-X parameters listed in Tab. I. In this example, the value of $\phi_{0}^{n}$ is set to $0.33^{\circ}$, equal to the satellite $3 \mathrm{~dB}$ azimuth antenna beamwidth. Four subswaths have been selected, for a total swath range coverage of $120 \mathrm{~km}$. The resulting parameters are listed in Tab. II. TerraSAR-X is able to steer the antenna in the azimuth direction within a range of $\pm 0.75^{\circ}$. Therefore, it can be seen that TerraSAR-X can in principle achieve TOPSAR $16 \mathrm{~m}$ azimuth resolution covering 4 subswaths, reaching the same coverage and resolution as the nominal TerraSAR-X ScanSAR product.

\section{STEERING ANGLE QUANTIZATION DESCRIPTION}

During a burst of the TOPSAR acquisition, the antenna beam is rotated from backward to forward in the flight direction. In electronic steered antennas, a continuous steering is often not feasible due to a limited angle storage table; therefore, a quantization in the steering law is present. The quantized angle has the typical step behavior and is analytically described as:

$$
\phi_{q}=\phi-\bmod (\phi, \Delta \phi)+\frac{\Delta \phi}{2}
$$

where $\Delta \phi$ is the angle quantization step. Such a quantization causes a modulation of the TOPSAR amplitude response. When (6) is inserted in (1), the resulting antenna gain suffers of amplitude modulation as illustrated in Fig. 1.

The angle quantization in TerraSAR- $X$ is equal to 0.006 degree. However, this is the angle difference between two close values of the 256 possible angles stored in the satellite memory. Another limit is imposed by the fact that only 125 values can be used in one data take, and this number has to

TABLE II

TERRASAR-X TOPSAR PARAMETERS

\begin{tabular}{|c|c|c|c|c|}
\hline & SS1 & SS2 & SS3 & SS4 \\
\hline Input parameters & & & & \\
\hline Mean look angle - [deg] & 28.464 & 30.426 & 32.075 & 34.086 \\
\hline Slant middle range - [km] & 591.8 & 604.6 & 616.5 & 632.5 \\
\hline PRF - [Hz] & 3558 & 3475 & 3836 & 3748 \\
\hline Azimuth resolution - [m] & 16 & 16 & 16 & 16 \\
\hline Calculated parameters & & & & \\
\hline Steering angle rate - [deg/s] & 3.2486 & 3.1796 & 3.1182 & 3.0393 \\
\hline DTAR - [dB] & -25.3 & -25.6 & -25.3 & -25.4 \\
\hline Burst time - [s] & 0.2649 & 0.2667 & 0.2684 & 0.2707 \\
\hline Dwell time - [s] & 0.08446 & 0.08630 & 0.0880 & 0.09028 \\
\hline Doppler bandwidth - [Hz] & 425 & 425 & 425 & 425 \\
\hline Maximum angle - [deg] & \pm 0.430 & \pm 0.424 & \pm 0.428 & \pm 0.411 \\
\hline
\end{tabular}

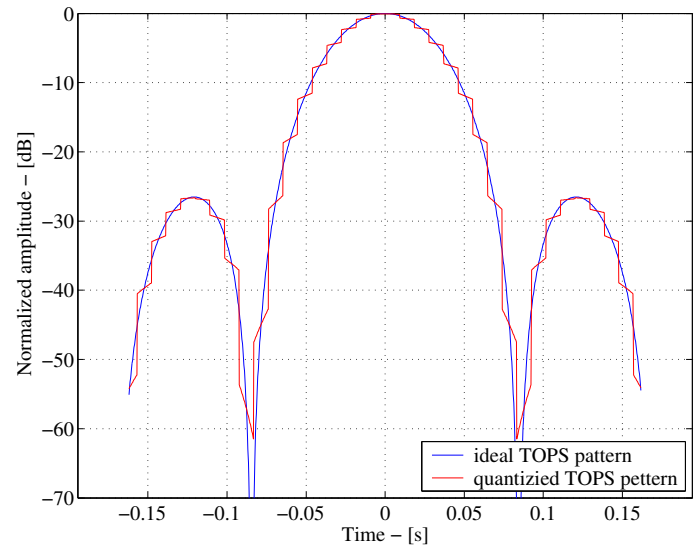

Fig. 1. Effects of the steering angle quantization on the TOPSAR antenna gain. The simulated quantization angle is $0.03^{\circ}$.

be shared for the whole number of subswaths [3]. By using the values of the maximum steering angles derived for the configuration of Tab. II, the resulting angle quantization is calculated to be approximately 0.03 degree. In Fig. 2, the resulting azimuth quantization imposed by the limited number of steering angles in TerraSAR-X is plotted as a function of the azimuth resolution and for different number of subswaths.

Another constraint on the quantization angle is imposed by the dwell time. In fact, in order to guarantee a uniform SNR and DTAR, every target has to be illuminated by the whole processed antenna beam. In turn, this means that the azimuth antenna steering angle has to change more than a certain number of times during the dwell time. Figure 3 shows the maximum angle quantization allowed as a function of the azimuth resolution for different numbers of azimuth beams (or angular steps) per target. The curves do not depend on the range.

\section{STEERING ANGLE QUANTIZATION EFFECTS}

In order to analyze the effects of the steering angle quantization of TerraSAR-X in TOPSAR configuration, a point

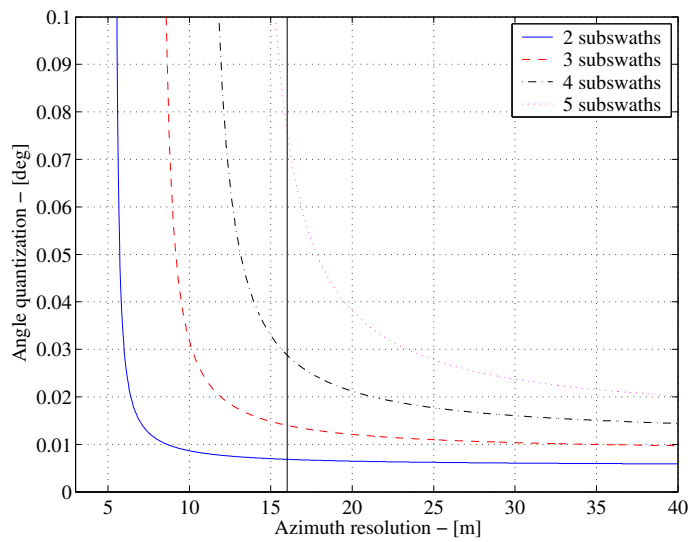

Fig. 2. Minimum angle quantization satisfying the constraint imposed by the TerraSAR-X limited number of steering angles per data take (125 angles) plotted as a function of the azimuth resolution. 


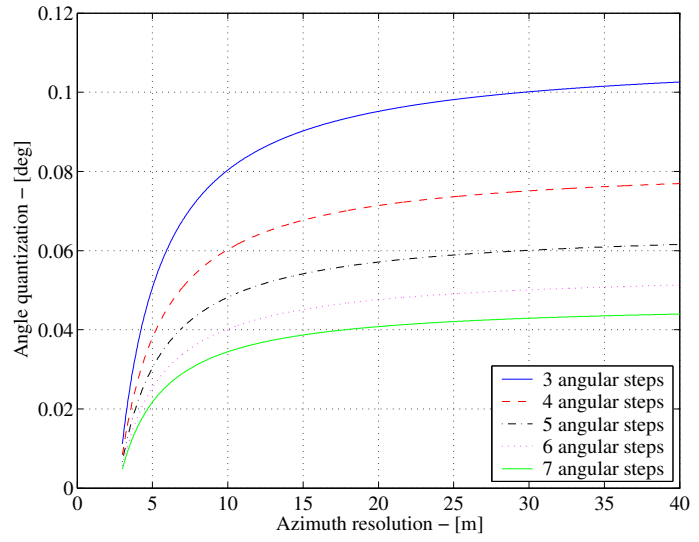

Fig. 3. Maximum angle quantization imposed by the uniform ambiguity noise constraint as a function of the azimuth resolution and for different values of the angular steps per target. The curves do not depend on the range.

target response has been simulated. The point target has been placed in the middle of the first subswath of Tab. II and an angle quantization of $0.03^{\circ}$ has been used. The simple spectral analysis approach has been used for the azimuth compression.

A quantized steering angle results in a quantized antenna pattern amplitude. Therefore, a periodic amplitude modulation is present in the unfocused azimuth response. The amplitude modulation is equal to the difference between the uniform and the quantized antenna gain; it distorts the focused azimuth response introducing spurious peaks.

For a first performance analysis, the amplitude error can be modelled as a sawtooth signal, defined as:

$$
x_{s t}\left(t, T_{q}\right)=A_{e} \sum_{n} \operatorname{rect}\left(\frac{t-n T_{q}}{T_{q}}\right) \cdot\left(t-n T_{q}\right)
$$

where $T_{q}=\Delta \phi / k_{n}$ is the period of the sawtooth signal. The model does not explicitly assume any weighting so that the resulting analytical analysis is already normalized to the TOPSAR peak response of the corresponding target. The advantage of using this model lies on the fact that it is easy to derive an analytical expression of its Fourier transform, whose amplitude is equal to:

$$
\left|X_{s t}(f)\right|=\frac{A_{e}}{\pi\left|f T_{q}\right|} \int \sum_{n \neq 0} \delta\left(f-\frac{n}{T_{q}}\right) \mathrm{d} f
$$

In a practical situation, the delta function is replaced by the sinc function due to the finite observation time. The term $A_{e}$ represents the maximum value of the error amplitude; this value approximately corresponds to the difference between the uniform and the quantized antenna gain calculated at the $3 \mathrm{~dB}$ point:

$$
A_{e}=\operatorname{sinc}^{2}\left(\frac{1}{2}\right)-\operatorname{sinc}^{2}\left(\frac{1}{2}-\frac{\Delta \phi}{2 \phi_{3 d B}}\right)
$$

The ideal TOPSAR response for the antenna pattern of Fig. 1, the quantized response and the bound of the analytical curve for the amplitude error (equation (8)) are plotted in Fig. 4. No windowing has been used during the azimuth

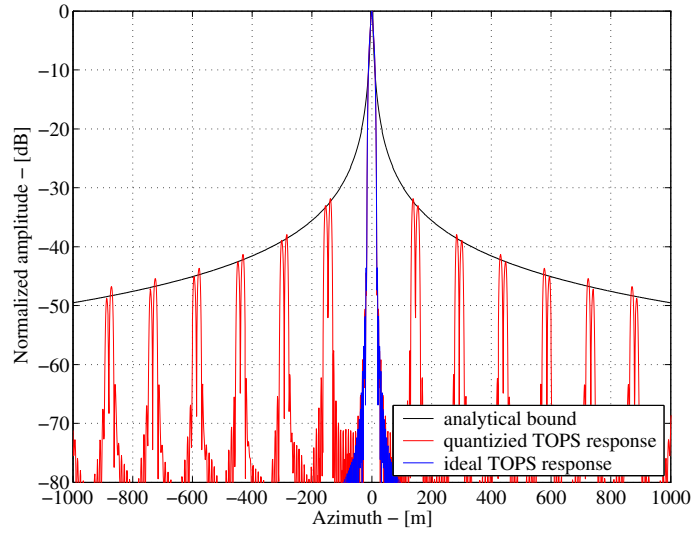

Fig. 4. TOPSAR response for the ideal uniform antenna (blue) and the quantized antenna gain case (red). In the picture, the error analytical bound is also plotted (black).

compression. When using windowing, the distortion peaks are decreased because the value of $A_{e}$ is reduced. An improvement of circa $5 \mathrm{~dB}$ in the spurious peak level is obtained with a Hamming window spanning the $3 \mathrm{~dB}$ integration angle, as shown in Fig. 5. Expression (8) can be rewritten in terms of the angle quantization in order to derive the level of the first (and maximum) resulting spurious peak:

$$
S_{q}(\Delta \phi)=\max \left|X_{s t}(f)\right|_{\Delta \phi}=\frac{A_{e}}{\pi}
$$

Equation (10) does not depend on the antenna steering rate, and therefore on the range, and is plotted in Fig. 6. The plot shows that, for practical values of the quantization angle to be used in TerraSAR-X TOPSAR applications, the resulting response distortion induced by the amplitude modulation is negligible.

\section{TERRASAR-X TOPSAR INTERFEROMETRY}

The performance achievable by an interferometric TOPSAR depends upon the capability to observe the same area with the

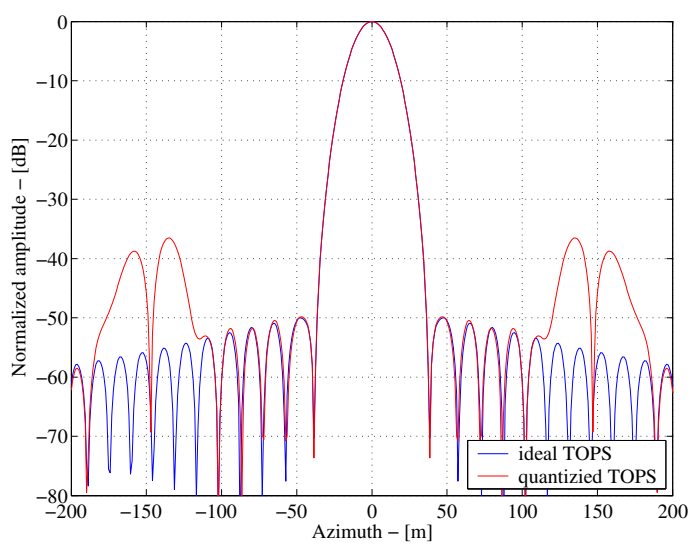

Fig. 5. TOPSAR response for the ideal and quantized case when the antenna pattern is modelled as a sinc squared and windowed with a Hamming window in the $3 \mathrm{~dB}$ beamwidth interval. 


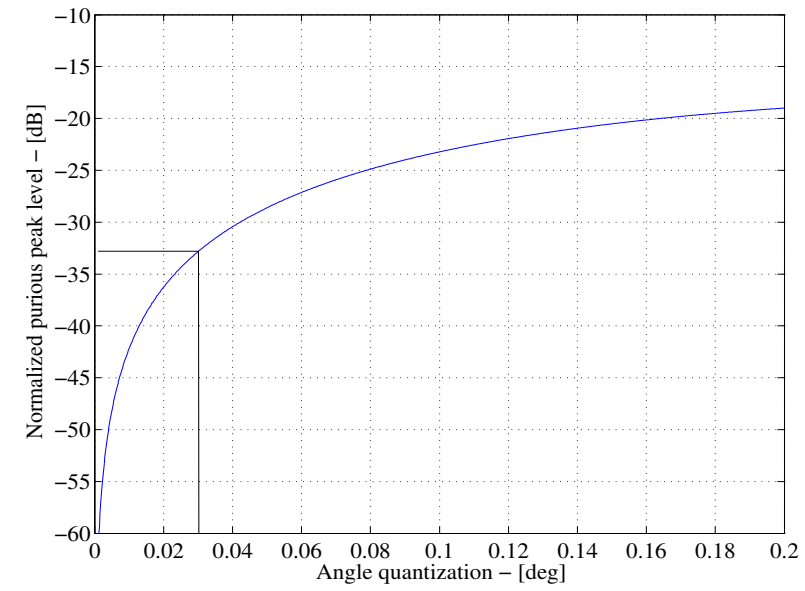

Fig. 6. Spurious peak level versus angle quantization. The curve is independent of the range distance and azimuth resolution.

same squint angle. A squint error $\delta \phi$ translates into a Doppler shift of:

$$
f_{\text {Dshift }}=\delta \phi \frac{2 v}{\lambda}
$$

The total squint error $\delta \phi$ is due to the contribution of both the antenna steering angle accuracy and the satellite alongtrack position accuracy, respectively $0.02^{\circ}$ and $20 \mathrm{~m}$ for TerraSAR-X. For a slant range of $590 \mathrm{~km}$, the latter translates into a squint angle error of $0.002^{\circ}$; therefore the maximum total squint angle error is $0.022^{\circ}$, leading to a maximum Doppler shift of approximately $167.5 \mathrm{~Hz}$. For the TerraSAR-X TOPSAR configuration (16 $\mathrm{m}$ azimuth resolution), the focused Doppler bandwidth $B_{D}$ is $425 \mathrm{~Hz}$; therefore the maximum relative interferometric resolution loss is:

$$
f_{\text {Dshift }} / B_{D}=\frac{167.5}{425}=0.39
$$

In terms of interferometric resolution loss TOPSAR is equivalent to ScanSAR. Finally, large Doppler centroids under which targets are observed at the edge of the TOPSAR bursts impose strong requirements in the coregistration accuracy.

\section{INVERSE TOPSAR}

The basic principle of TOPSAR is the shrinking of the antenna pattern obtained by steering the antenna in the opposite direction as for Spotlight. The concept is analytically expressed by (1). For resolution, SNR and DTAR performance, the driving parameter is $\alpha$, namely its absolute value since the antenna gain is symmetric. In principle, the same absolute value of $\alpha$ can be obtained steering the antenna in the same direction as in Spotlight, with a steering angle rate $k_{i \phi}$. In the rest of the analysis we use always positive values for both $k_{\phi}$ and $k_{i \phi}$. The value of $k_{i \phi}$, relative to a TOPSAR $k_{\phi}$, can be found by imposing:

$$
\left|1+\frac{k_{\phi} R_{0}}{v}\right|=\left|1-\frac{k_{i \phi} R_{0}}{v}\right|
$$

resulting in:

$$
k_{i \phi}=\frac{2 v}{R_{0}}+k_{\phi}
$$

The value of $k_{i \phi}$ is higher than the corresponding $k_{\phi}$, because of the opposite contribution of the platform velocity. Imposing the same continuous coverage constraint as for conventional TOPSAR, the inverse TOPSAR time line can be calculated by solving a similar set of equation as for conventional TOPSAR. The result is the same time line. Therefore, the two modes share the same time line, coverage properties, SNR and DTAR performance.

The main difference between TOPSAR and inverse TOP$\mathrm{SAR}$ is that the latter requires a higher antenna steering rate, and being the burst time the same, a higher antenna steering capability. Similar concepts have been found also in [4].

An advantage for TerraSAR-X in using the inverse TOPSAR acquisition mode relays on the fact that the same data commanding as for Spotlight can be used; the TerraSAR-X commanding for steering the antenna from forth to back, in the way it is currently implemented, is more efficient from an uplink point of view because it just requires the starting and final angle and the number of steps. Steering the antenna from back to forth requires instead the commanding of every single azimuth angle. However, conventional TOPSAR is in general preferable to inverse TOPSAR in electronic steering antenna satellites, as TerraSAR-X.

Differently, in mechanic steering antenna platform, where an instantaneous switching of the point angle is not possible, a combination of conventional TOPSAR and inverse TOPSAR can still allow the sensor to have the same coverage as with electronic steering devices, provided that the antenna is able to periodically rotate with different rates depending on the rotation direction.

\section{CONCLUSION AND FUTURE WORK}

The paper has presented investigations carried out for the implementation of TOPSAR acquisition mode with TerraSAR-X. The effects of the quantization of the steering angle are negligible. The TerraSAR-X steering capability allows TOPSAR operations with the same coverage and resolution as the nominal ScanSAR. Several data takes with TerraSAR$\mathrm{X}$ TOPSAR and inverse TOPSAR configuration have been planned. The data will be processed with the processor developed in DLR [5] and compared with ScanSAR images of the same areas.

\section{REFERENCES}

[1] A. M. Guarnieri and C. Prati, "ScanSAR focusing and interferometry," IEEE Trans. on Geoscience and Remote Sensing, vol. 34, no. 4, pp. 10291038, July 1996.

[2] F. D. Zan and A. M. Guarnieri, "Topsar: terrain observation by progressive scan," IEEE Trans. Geosci. Remote Sensing, vol. 44, no. 9, pp. 23522360, Sept. 2006.

[3] TX-IOCS-DD-4402-VOL-06, "TerraSAR-X Instrument Table Generator Design Document."

[4] D. D'Aria and D. Giudici, "Tops-sar system analysis and optimization," in GMES Sentinel-1 project, ESTEC Presentation, The Netherlands, Mar. 2006.

[5] P. Prats, R. Scheiber, J. Mittermayer, A. Meta, and A. Moreira, "A SAR Processing Algorithm for TOPS Imaging Mode Based on Extended Chirp Scaling," in Proc. IEEE Int. Geoscience and Remote Sensing Symp. IGARSS'07, Barcelona, Spain, July 2007. 